\title{
Validation of single nucleotide polymorphisms associated with milk production traits in dairy cattle
}

\author{
A. J. Chamberlain, ${ }^{\star 1}$ B. J. Hayes, ${ }^{\star}$ K. Savin, ${ }^{\star}$ S. Bolormaa, ${ }^{\star}$ H. C. McPartlan, ${ }^{\star 2}$ P. J. Bowman, ${ }^{\star}$ \\ C. Van Der Jagt, $\uparrow$ S. MacEachern, $\dagger^{3}$ and M. E. Goddard ${ }^{\star} \dagger$ \\ ${ }^{*}$ Biosciences Research Division, Department of Primary Industries Victoria, Bundoora, 3083, Australia \\ †Land and Food Resources, University of Melbourne, Parkville, 3052, Australia
}

\begin{abstract}
Single nucleotide polymorphism (SNP) associations with milk production traits found to be significant in different screening experiments, including SNP in genes hypothesized to be in gene pathways affecting milk production, were tested in a validation population to confirm their association. In total, $423 \mathrm{SNP}$ were genotyped across 411 Holstein bulls, and their association with 6 milk production traits-Australian Selection Index (indicating the profitability of an animal's milk production), protein, fat, and milk yields, and protein and fat composition-were tested using single SNP regressions. Seventy-two SNP were significantly associated with one or more of the traits; their effects were in the same direction as in the screening experiment and therefore their association was considered validated. An over-representation of SNP (43 of the 423) on chromosome 20 was observed, including a SNP in the growth hormone receptor gene previously published as having an association with protein composition and protein and milk yields. The association with protein composition was confirmed in this experiment, but not the association with protein and milk yields. A multiple SNP regression analysis for all SNP on chromosome 20 was performed for all 6 traits, which revealed that this mutation was not significantly associated with any of the milk production traits and that at least 2 other quantitative trait loci were present on chromosome 20 .
\end{abstract} Key words: validation, single nucleotide polymorphism, milk production

\section{INTRODUCTION}

Genome-wide association studies (GWAS) interrogate the entire genome for associations between DNA

\footnotetext{
Received September 6, 2010.

Accepted May 7, 2011.

${ }^{1}$ Corresponding author: amanda.chamberlain@dpi.vic.gov.au

${ }^{2}$ Current address: Department of General Practice, University of Melbourne, Carlton, 3053, Australia.

${ }^{3}$ Current address: Cobb-Vantress Inc., Siloam Springs, AR 72761.
}

variants such as SNP and a phenotype. These associations determine the location of QTL, loci involved in the control of quantitative traits. Genome-wide association studies have been made possible in cattle through the sequencing of the bovine genome and the discovery of hundreds of thousands of SNP (Bovine HapMap Consortium, 2009; Elsik et al., 2009). This has resulted in the development of commercial SNP chips and rapid, large-scale genotyping of common SNP across large populations. Association with the phenotype is often determined by carrying out a statistical test for each SNP in turn. Such multiple testing requires very high significance thresholds to avoid false positives. However, such demanding significance thresholds would result in many true effects not being detected, whereas a relaxed threshold would lead to the acceptance of many false positives (Lander and Kruglyak, 1995; Weller et al., 1998). Lander and Kruglyak (1995) suggested that it was not possible to distinguish between false positives and true associations. Instead, they suggested a classification of genome-wide statistical significance into suggestive, significant, highly significant, and confirmed linkage. Benjamini and Hochberg (1995) proposed controlling the false discovery rate (FDR) as an alternative to controlling the experiment-wide error rate. Similar methods have been developed by Mosig et al. (2001) and Storey and Tibshirani (2003). However, the most reliable way of testing significance is to validate the SNP effect in another population (Lander and Kruglyak, 1995; Visscher, 2008), because the probability of a SNP being significant in two populations by chance is small, particularly if two or more validation populations of different breeds are used (Karlsson et al., 2007).

An alternative approach to finding polymorphisms associated with milk production traits has been the candidate gene approach, in which individual genes are selected as candidates based on their known function. Although this approach has met with limited success, more promising recent studies have investigated large numbers of candidates based on gene expression studies and identified those that showed differential expression, 
or fell into gene pathways with links to trait physiology where multiple genes were differentially expressed (Naukkarinen et al., 2010). Of course, associations detected in such experiments still require validation in independent populations for the reasons already described.

In this paper, we first report the results of 3 GWAS for milk production traits in Australian dairy cattle. The key trait of interest for most of these studies was the Australian Selection Index (ASI), an index that has positive weightings on protein yield and, to a lesser extent, fat yield, while having a negative weight on milk yield. This index indicates the profitability of an animal's milk production (Pryce et al., 2009). The GWAS included a 10,000-SNP bull experiment (10K), with approximately 10,000 SNP genotyped on 384 Holstein bulls selected to be extreme for ASI; a 25,000-SNP cow experiment $(\mathbf{2 5 K})$, with approximately 25,000 SNP genotyped on 200 Holstein cows selected to be extreme for ASI; a 10,000-SNP bull and cow experiment $\left(\mathbf{1 0 K} \_\mathbf{b}+\mathbf{c}\right)$, which combined the above animals (384 bulls and 200 cows) and included the 9,919 SNP that were within both the $10 \mathrm{~K}$ and $25 \mathrm{~K}$ experiments. We also report on the results of a candidate gene study (CGS), which involved genotyping the above 384 Holstein bulls on SNP selected from candidate genes located within regions largely associated with milk production traits from the above $10 \mathrm{~K}$ experiment. In total, $617 \mathrm{SNP}$ were genotyped from 26 regions of the bovine genome. Some SNP were discovered from in-house sequencing of candidate genes, and others were selected from public SNP databases. Finally, the significant SNP from these experiments were tested in a validation population of 411 Holstein bulls. The validated SNP will contribute to panels of markers for genomic selection and reveal some of the genes involved in variation in milk production traits.

\section{MATERIALS AND METHODS}

\section{SNP Screening Experiments}

$10 K$ Experiment. The DNA samples were extracted from semen of 384 Holstein bulls selected to be have extreme ASI phenotypes, using the Gentra Puregene Tissue Kit (Qiagen, Valencia, CA). They were genotyped by the Affymetrix SNP assay service using the Genechip Bovine Mapping 10K SNP kit (Affymetrix, Santa Clara, CA). This kit assayed 9,919 SNP in a single reaction (Hardenbol et al., 2003). In total, 847 monomorphic SNP were removed from the data set, and 9,072 SNP were retained for analysis.

Australian Breeding Values (ABV) for protein, fat, and milk yields, and protein and fat composition were provided by the Australian Dairy Herd Improvement Scheme (ADHIS). Because a bull's breeding value includes both pedigree information and daughter phenotypic production (adjusted for fixed effects), the risk exists that SNP would be selected based on pedigree information rather than phenotype. Therefore, deregressed solutions removing the contribution of information from relatives were calculated for each sire. The methodology for this deregression is described by Pryce et al. (2010a), but for completeness, it is also described in the Appendix. As the phenotypic and genetic correlations between traits are the same (Pryce et al., 2010b), ASI was then calculated using the same weightings as would be used with breeding values; that is, 3.8 protein yield +0.9 fat yield -0.048 milk yield. If these correlations were different, the weightings would have needed to be adjusted.

ASReml (Gilmour et al., 2001) was used to conduct single SNP regressions using the following model:

$$
y_{i}=\mu+a_{i}+\beta x+e_{i},
$$

where $y$ is the deregressed breeding value for animal $i$, $a_{i}$ is a polygenic breeding value, where $a \sim N\left(0, \mathbf{A} \sigma_{A}^{2}\right)$, with $\mathbf{A}$ being a matrix of additive genetic relationships among the population, $\sigma_{A}^{2}$ is the additive genetic variance not explained by the markers, $\beta$ is the effect of the SNP alleles $x$, coded as 0,1 , or 2 for homozygote allele 1 , heterozygote, homozygote allele 2 , respectively, carried by animal $i$, and $e$ is the error. Each SNP was tested for association with ASI, protein, fat, and milk yields, and protein and fat composition. The SNP were taken as significant if the $F$-value exceeded 10.51 (i.e., $P<0.001)$ and SNP effects estimated as the difference between allele 1 and 2 .

25 K Experiment. The DNA samples of $200 \mathrm{Hol}-$ stein cows, selected to have extreme ASI phenotypes, were extracted from whole blood using the Gentra Puregene Blood Kit (Qiagen).They were genotyped with the Affymetrix Genechip Bovine Mapping 25K SNP kit (Affymetrix) by Cigene (Ås, Norway). This kit assays 25,069 SNP in a single reaction (Hardenbol et al., 2003). Then, 2,070 SNP were removed because they were monomorphic; 3,082 polymorphic SNP were removed because they had a rare allele frequency $<5 \%$; 1,276 SNP were removed because they had more than $20 \%$ of their genotypes missing; and 550 SNP failed a Hardy-Weinberg equilibrium (HWE) test. Hardy Weinberg equilibrium was assessed for each family because it was assumed that if a sire was heterozygous for a SNP with a rare allele, the progeny from that sire family are unlikely to deviate from HWE. A sire family was judged to fail HWE if $P<0.001$; SNP failed the 
HWE test if more than 2 sire families were $P<0.001$. In total, 18,990 SNP were retained for analysis. Again, deregressed breeding values were used as phenotypes to conduct single SNP regressions. The methodology for the deregression of cow breeding values can be found in the Appendix.

$\boldsymbol{C G S}$. From the above 10K experiment, $26 \mathrm{SNP}$ were significantly associated $(F>11)$ with ASI. At the time, these SNP were mapped to bovine genome build 3.1 and later build 4.0 (supplementary Table 1, available at http://www.journalofdairyscience.org/). These 26 regions of bovine genome build 3.1 and the homologous regions of the human genome were searched for candidate genes hypothesized, based on published functional information predominantly from human and mouse data on NCBI (http://www.ncbi.nlm.nih.gov/) and Ensembl (http://www.ensembl.org/index.html) websites, to be in pathways affecting milk production traits. Two hundred twenty-one candidate genes were identified within these 26 regions or their homologous regions within the human genome. An additional 43 candidates, selected in the same way, were included from the remainder of the bovine genome. In total, 264 candidate genes were identified (Supplementary Table 2, available at http://www.journalofdairyscience.org/), and 617 new SNP (not already on the 10,000 SNP chip) within or close to these were selected for genotyping across the same 384 Holstein bulls described above. Some SNP were discovered from in-house sequencing of candidate genes, and others were selected from public SNP databases. Although selected based on their positioning to bovine genome build 3.1, sometimes using homologous mapping to the human genome, these SNP were mapped to bovine genome build 4.0 by using BLAST (http://blast.ncbi.nlm.nih.gov/Blast. cgi). This resulted in some SNP not mapping to the correct locations within bovine genome build 4.0. The SNP were divided among 13 probe pools and genotyped using Applied Biosystems SNPLEX chemistry on the ABI 3730xl DNA Analyzer (Applied Biosystems, San Diego, CA). Genotype calling was performed using Genemapper software. In total, 519 of the 617 SNP were polymorphic and genotypes could be read with confidence. Data were checked for inconsistencies and pedigree errors using an in-house database. Deregressed breeding values were used to conduct single SNP regressions (same as in the 10K study) and SNP were taken as significant if the $F$-value exceeded $3.89(P<0.05)$.

\section{SNP Validation Experiment}

In total, $423 \mathrm{SNP}$ were genotyped across 411 Holstein bulls not previously genotyped for these SNP. The 411 bulls were from a breeding company's (Genetics Aus- tralia, Bacchus Marsh, Victoria, Australia) 2001-2003 progeny test teams. The 411 DNA samples were extracted from semen using the Gentra Puregene Tissue Kit (Qiagen), and the 423 SNP were divided among 9 probe pools and genotyped using Applied Biosystems SNPLEX chemistry on the ABI 3730xl DNA Analyzer. Genotype reading was performed using Genemapper software. Data were checked for inconsistencies and pedigree errors using an in house database. The ABV for protein, fat, and milk yields, and protein and fat composition were provided by ADHIS, and were deregressed for each sample, and the ASI were calculated (same as in the 10K study).

\section{Statistical Analysis}

Single SNP Regressions. ASReml was used to conduct single SNP regressions using the model described for the SNP screening populations. Each SNP was tested for 6 traits; SNP were taken as significant if the $F$-value exceeded $3.89(P<0.05)$ and SNP effects estimated as the difference between allele 1 and 2 .

False Discovery Rates. False discovery rates (FDR; the proportion of tests likely to be false positives) were calculated as follows: $F D R=\left(\frac{m \times \operatorname{Pr}(>0)}{R}\right)$, as described by Benjamini and Hochberg (1995), where $m$ is the total number of tests, $R$ is the number of significant tests, and $\operatorname{Pr}(>0)$ is equal to the significance threshold applied.

An alternative FDR $\left(\mathbf{F D R}_{\mathbf{a}}\right)$ was calculated as $1-2\left(s-\frac{1}{2}\right)$, where $s$ is the observed proportion of significant SNP with effects in the same direction as in the screening population. This is the FDR of the significant SNP, $P<0.05$, with SNP effects in the same direction as the screening experiment.

Multiple SNP Analysis of Chromosome 20. Ten percent (43 of 423) of the SNP in the validation data set were on chromosome 20, which represents only $3 \%$ of the bovine genome; therefore, an over-representation of SNP on this chromosome was observed in our study. This seems to stem from the fact that $17 \%$ of the SNP in the CGS study were on chromosome 20, a result of the number of regions on chromosome 20 that contained QTL from the 10K GWAS and the number of candidate genes selected from those regions. However, to determine if one or multiple QTL were present on this chromosome, a multiple SNP regression analysis was performed on the SNP on this chromosome, including a published mutation within the growth hormone receptor (GHR) gene (Blott et al., 2003) associated with protein composition and protein and milk yields. 
Missing genotypes varied for each SNP (6-132). For single SNP analysis, these were treated as missing values; however, this did not greatly affect the data set, because the proportion of missing values was small in the majority of cases. However, when combined across SNP, the number of missing genotypes resulted in large numbers of animals being excluded from the analysis, thus reducing the power of the experiment. To enable a multiple SNP regression analysis, missing genotypes were imputed. This was done with fastPHASE (Scheet and Stephens, 2006). All SNP were fitted in sequential multiple SNP regressions for all 6 traits to determine if more than one QTL existed. First, each SNP was fitted along with the most significant SNP, across all traits, from the single SNP regression in a 2-SNP regression. Then, each SNP was fitted along with the most significant SNP, across all traits, from the single SNP regression and the 2-SNP regression in a 3-SNP regression, and so on, until no more SNP were significant. The SNP were taken as significant if the $F$-value exceeded 3.89 (i.e., $P<0.05$ ). ASReml was used to conduct the multiple SNP regressions using the following model:

$$
y_{i}=\mu+a_{i}+\beta_{1} x_{1}+\beta_{2} x_{2} \ldots+e_{i},
$$

where $y_{i}$ is the deregressed breeding value for animal $i$; $a_{i}$ is a polygenic breeding value, where $a \sim N\left(0, \mathbf{A} \sigma_{A}^{2}\right)$, with $\mathbf{A}$ being a matrix of additive genetic relationships among the population, $\sigma_{A}^{2}$ is the additive genetic variance not explained by the markers; $\beta_{1}$ is the effects of the first SNP alleles, $x_{1}$, coded as 0,1 , or 2 for homozygote allele 1 , heterozygote, and homozygote allele 2 , respectively; and $\beta_{2}$ is the effects of the second SNP alleles, $x_{2}$, also coded as 0,1 , or 2 , carried by animal $i$; and $e_{i}$ is the error.

\section{RESULTS}

\section{SNP Screening Experiments}

Table 1 summarizes the results from each of the SNP screening experiments and presents the number of SNP genotyped and tested for each experiment. These differ because some SNP were excluded from the analysis in each experiment based on quality criteria; for example, low minor allele frequencies and call rates deviating from HWE. Also presented are the number of significant SNP and the FDR at a significance threshold of $P<0.001$ for each of the 6 traits tested (ASI, protein yield, protein composition, fat yield, fat composition, and milk yield).

\section{SNP Selection}

In total, $199 \mathrm{SNP}$ were selected from the 10K experiment; these were found to be most highly associated $(P<0.008)$ with ASI. One hundred thirty-one SNP passed assay design. One hundred forty-five SNP from the CGS experiment were selected because they were found to be significantly $(P<0.05)$ associated with ASI, protein, fat, and milk yields, and protein or fat composition; 141 passed assay design.

Seventy-seven SNP were selected from the $25 \mathrm{~K}$ experiment because they were significantly associated with ASI $(P<0.001)$ and at least one other production trait $(P<0.008) ; 69$ passed assay design.

One hundred twelve SNP were selected from the 10K_b+c experiment because they were significantly

Table 1. The number of SNP genotyped, tested, and significant at $P<0.001$ and the false discovery rate (FDR) for the 6 traits from each of the SNP screening experiments ${ }^{\mathrm{T}}$

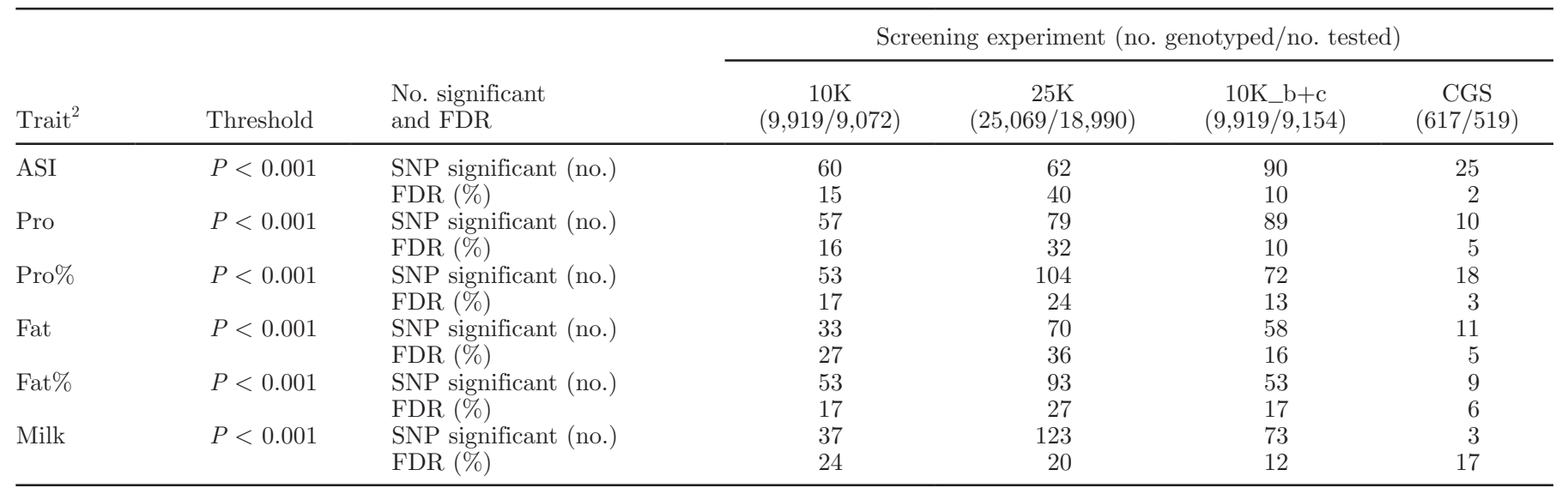

${ }^{1} 10 \mathrm{~K}=10,000$ SNP bull experiment; $25 \mathrm{~K}=$ the 25,000 SNP cow experiment; $10 \mathrm{~K} \_\mathrm{b}+\mathrm{c}=$ the 10,000 SNP cow and bull experiment; CGS $=$ candidate gene study.

${ }^{2} \mathrm{ASI}=$ Australian Selection Index (ASI); Pro = protein yield; Pro $\%=$ protein composition; Fat $=$ fat yield; Fat $\%=$ fat composition; and Milk $=$ milk yield. 
associated with ASI $(P<0.001)$ and at least one other production trait $(P<0.008)$; 103 passed assay design.

Some SNP were selected from multiple experiments; therefore, 423 SNP in total were selected.

\section{SNP Validation Experiment}

Four hundred twenty-three SNP (including 176 from the GWAS and 113 from the CGS experiment) found to be significant in at least one of the screening experiments were genotyped across the 411 animals. Two hundred eighty-nine of these were polymorphic and passed genotype quality checks and were tested for all 6 traits. Using all SNP in the validation experiment and then only SNP significant in the screening experiments, the number of SNP tested, the number significant $(P<$ 0.05 ), along with FDR for all 6 traits, are presented in Table 2. Also presented are the numbers of SNP that are both significant and have effects in the same direction in both the reference and validation sets. These SNP were considered to be validated.

Table 3 contains a list of the 72 validated SNP and their locations within the bovine genome (btau4.0). Another large GWAS has recently been conducted in Australian dairy cattle using 51,386 SNP (Pryce et al., 2010a). Therefore, also presented in Table 3 is the distance to the most significant $(P<0.001) \mathrm{SNP}$, for the same trait and within $5 \mathrm{Mb}$, from that $50 \mathrm{~K}$ data set.

Of the $67 \mathrm{SNP}$ with known positions, 56 were within $5 \mathrm{Mb}$ of a significant SNP from the $50 \mathrm{~K}$ experiment (Pryce et al., 2010a). When the 50K SNP were assigned to $10-\mathrm{Mb}$ intervals, $96 \%$ of those intervals contained at least one SNP significant for the current experiment, for at least one trait. Therefore, 56 of 67 validated SNP being within $5 \mathrm{Mb}$ of a significant SNP from the $50 \mathrm{~K}$ experiment is fewer than expected (i.e., $96 \%$ of 67 is $64)$.

The correlation between SNP effects for ASI and protein composition for all SNP in the validation data set was 0.21 . However, the same correlation when including only significant SNP was 0.9. This correlation is high, meaning that the effects for both traits are either positive or negative, and the magnitude of the effects is similar.

\section{Chromosome 20 Multi-SNP Analysis}

Blott et al. (2003) published a mutation within the GHR gene that was associated with protein composition, and protein and milk yields. Figure 1 presents the results of the single SNP regression analysis for all SNP on chromosome 20 for protein composition and protein and milk yields. It shows that the published GHR mutation (the enlarged solid data points in each series at $33 \mathrm{Mb}$ ) is only associated with protein composition in our population; no significant association was observed with milk and protein yields. However, another SNP at $39 \mathrm{Mb}$ is associated with milk and protein yields. A multiple SNP regression analysis was performed across all traits to determine if all SNP were detecting the association with the published GHR mutation or whether multiple QTL exist on this chromosome.

Table 2. The number of SNP tested, significant $(P<0.05)$, the false discovery rate (FDR) at $P<0.05$, the number and proportion of SNP significant and in the same direction as the screening experiment, and $\mathrm{FDR}_{\mathrm{a}}{ }^{1}$ for the 6 traits from the SNP validation experiment, differentiating between all SNP and only those SNP significant in the screening experiment for that trait

\begin{tabular}{|c|c|c|c|c|c|c|}
\hline \multirow[b]{2}{*}{ Item } & \multicolumn{6}{|c|}{ Trait $^{2}$} \\
\hline & ASI & Pro & Pro $\%$ & Fat & Fat $\%$ & Milk \\
\hline \multicolumn{7}{|l|}{ All SNP } \\
\hline Tested (no.) & 289 & 289 & 289 & 289 & 289 & 289 \\
\hline Significant $P<0.05$ (no.) & 35 & 23 & 47 & 25 & 38 & 28 \\
\hline $\operatorname{FDR}(\%)$ & (41) & $(63)$ & $(31)$ & $(58)$ & $(38)$ & $(52)$ \\
\hline $\begin{array}{l}\text { Significant and in same direction (no.) } \\
\operatorname{FDR}_{\mathrm{a}}(\%)\end{array}$ & $\begin{array}{l}25 \\
(57)\end{array}$ & $\begin{array}{c}7 \\
(100)\end{array}$ & $\begin{array}{c}32 \\
(64)\end{array}$ & $\begin{array}{c}14 \\
(88)\end{array}$ & $\begin{array}{c}23 \\
(79)\end{array}$ & $\begin{array}{l}16 \\
(86)\end{array}$ \\
\hline \multicolumn{7}{|l|}{ SNP significant ${ }^{3}$ in screening experiment } \\
\hline Tested (no.) & 226 & 193 & 97 & 171 & 42 & 104 \\
\hline Significant $P<0.05$ (no.) & 28 & 13 & 27 & 15 & 18 & 10 \\
\hline FDR $(\%)$ & $(40)$ & $(74)$ & (18) & $(57)$ & $(12)$ & $(52)$ \\
\hline $\begin{array}{l}\text { Significant and in same direction (no.) } \\
\operatorname{FDR}_{\mathrm{a}}(\%)\end{array}$ & $\begin{array}{l}20 \\
(57)\end{array}$ & $\begin{array}{c}4 \\
(100)\end{array}$ & $\begin{array}{c}20 \\
(52)\end{array}$ & $\begin{array}{l}10 \\
(67)\end{array}$ & $\begin{array}{c}13 \\
(56)\end{array}$ & $\left.\begin{array}{c}6 \\
(80)\end{array}\right)$ \\
\hline
\end{tabular}

${ }^{1}$ False discovery rate $\mathrm{a}=$ the rate of false discoveries when SNP must be both significant and have effects in the same direction in 2 data sets.

${ }^{2}$ ASI $=$ Australian Selection Index; Pro $=$ protein yield $;$ Pro $\%=$ protein composition; Fat $=$ fat yield $;$ Fat $\%=$ fat composition; and Milk = milk yield

${ }^{3}$ Genome-wide association study SNP significant at $P<0.001$, candidate gene study SNP significant at $P<$ 0.05 . 
Table 3. Locations, within Btau4.2 and UMD3.1 genome builds (both available at http://www.ncbi.nlm.nih.gov/mapview/map_search. cgi?taxid=9913), of validated SNP with the refSNP entry from NCBI dbSNP database, the traits the SNP affected, whether the SNP was selected from the candidate gene study (CGS) and the distance (bp) between the SNP and the most significant SNP $(P<0.001)$, within $5 \mathrm{Mb}$, from the $50 \mathrm{~K}$ experiment

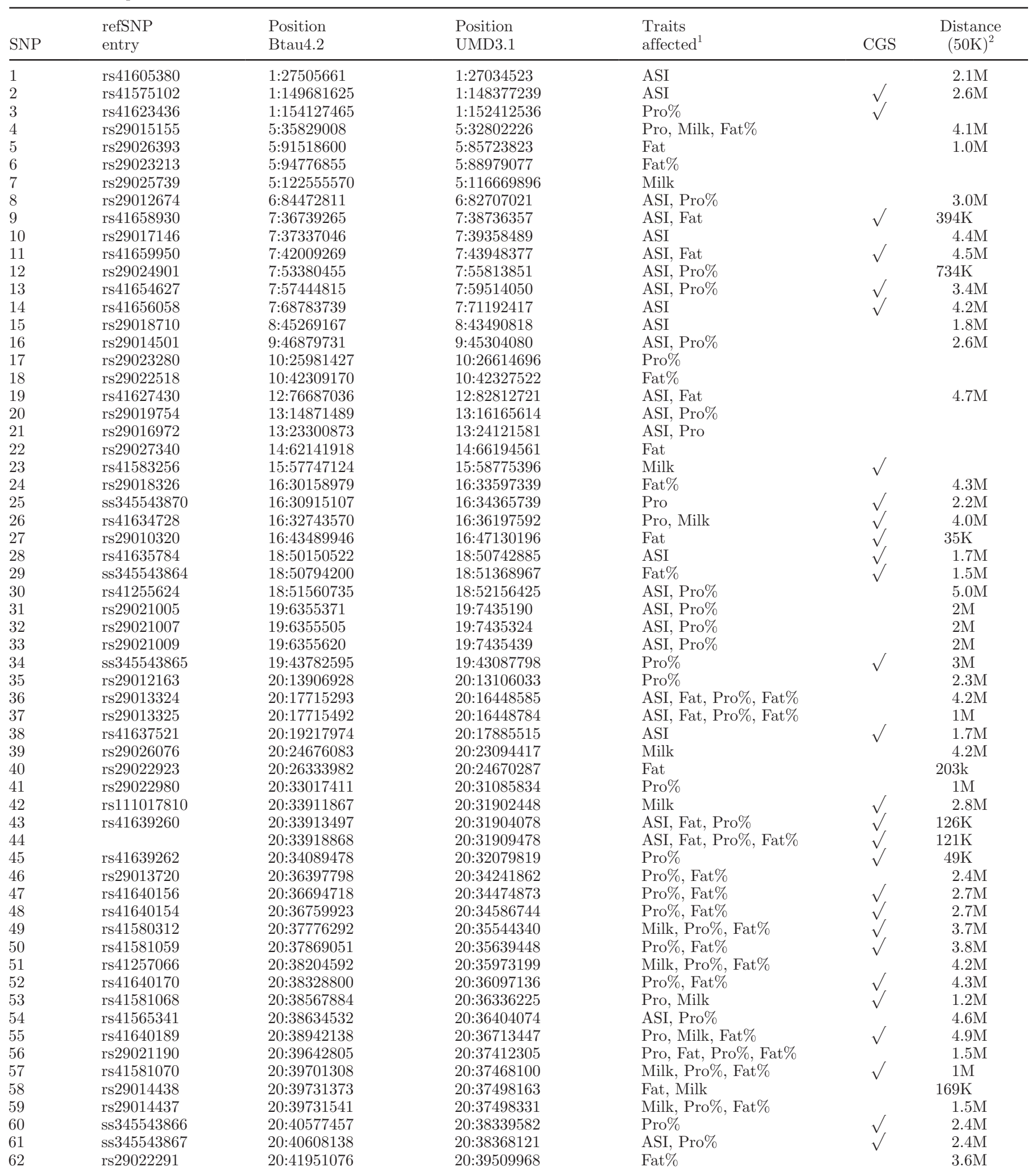

Continued 
Table 3 (Continued). Locations, within Btau4.2 and UMD3.1 genome builds (both available at http://www.ncbi.nlm.nih.gov/mapview/ map_search.cgi?taxid=9913), of validated SNP with the refSNP entry from NCBI dbSNP database, the traits the SNP affected, whether the SNP was selected from the candidate gene study (CGS) and the distance (bp) between the SNP and the most significant SNP $(P<0.001)$, within $5 \mathrm{Mb}$, from the $50 \mathrm{~K}$ experiment

\begin{tabular}{|c|c|c|c|c|c|c|}
\hline SNP & $\begin{array}{l}\text { refSNP } \\
\text { entry }\end{array}$ & $\begin{array}{l}\text { Position } \\
\text { Btau4.2 }\end{array}$ & $\begin{array}{l}\text { Position } \\
\text { UMD3.1 }\end{array}$ & $\begin{array}{l}\text { Traits } \\
\text { affected }^{1}\end{array}$ & CGS & $\begin{array}{c}\text { Distance } \\
(50 \mathrm{~K})^{2}\end{array}$ \\
\hline 63 & rs 29021045 & 23:30435849 & $23: 30163206$ & ASI, Pro & & \\
\hline 64 & rs 29027305 & $23: 37513882$ & $23: 36926025$ & ASI, Pro $\%$ & & $2.2 \mathrm{M}$ \\
\hline 66 & rs 29023528 & $26: 37220382$ & $26: 37034154$ & Fat, Fat\% & & $5 \mathrm{M}$ \\
\hline 67 & rs29018037 & $29: 38888724$ & $29: 37721984$ & Pro $\%$, Fat $\%$ & & $4.1 \mathrm{M}$ \\
\hline 68 & rs81111878 & & & Pro, Milk & $\sqrt{ }$ & \\
\hline 72 & rs 29018241 & & & ASI, Fat & & \\
\hline
\end{tabular}

${ }^{1}$ ASI $=$ Australian Selection Index Pro = protein yield; Pro $\%=$ protein percentage $;$ Fat $=$ fat yield Fat $\%=$ fat percentage; Milk = milk yield ${ }^{2}$ Pryce et al. (2010a).

The multiple SNP regression analysis across all 6 traits resulted in 14 SNP being significant. The most significant SNP in the single-trait analysis was the GHR SNP published by Blott et al. (2003). In addition, 2 SNP equally significant for protein composition were identified in the 2-SNP analysis. These SNP, located at 39,701,308 bp (rs41581070) and 39,731,541 bp (rs29014437), were highly correlated $\left(\mathrm{r}^{2}=1\right)$ and in perfect linkage disequilibrium; therefore, only one (located at 39,701,308 bp) was included in subsequent multi-SNP analyses. Other SNP within the remaining 13 SNP were highly correlated (Table 4). Because of these high correlations, the SNP set was reduced to 6 by excluding (starting with the last SNP fitted in the model) those that had $\mathrm{r}^{2}>0.4$ with another SNP within the set of 13 . Thus, SNP 13 (rs29016210, 13th in the model) was correlated with SNP 7 (rs41580312); SNP 7 (rs41580312, 12th in the model) with SNP 9 (rs41257066); SNP 5 (11th in the model) with SNP 6 (Blott et al., 2003 mutation); SNP 10 (rs41640170, 10th in the model) with SNP 9 (rs41257066); SNP 11 (rs29021190, 7th in the model) with SNP 9 (rs41257066); SNP 8 (rs41581059, 5th in the model) with SNP 12 (rs41581070); and SNP 1 (rs29017462, 4th in the model) with SNP 2 (rs29013325). The final 6 SNP multiple regression analysis results are presented in Table 5. Looking at the SNP effects in Table 5, SNP at $17,715,492,24,676,083$, and $26,333,982$ bp all appear

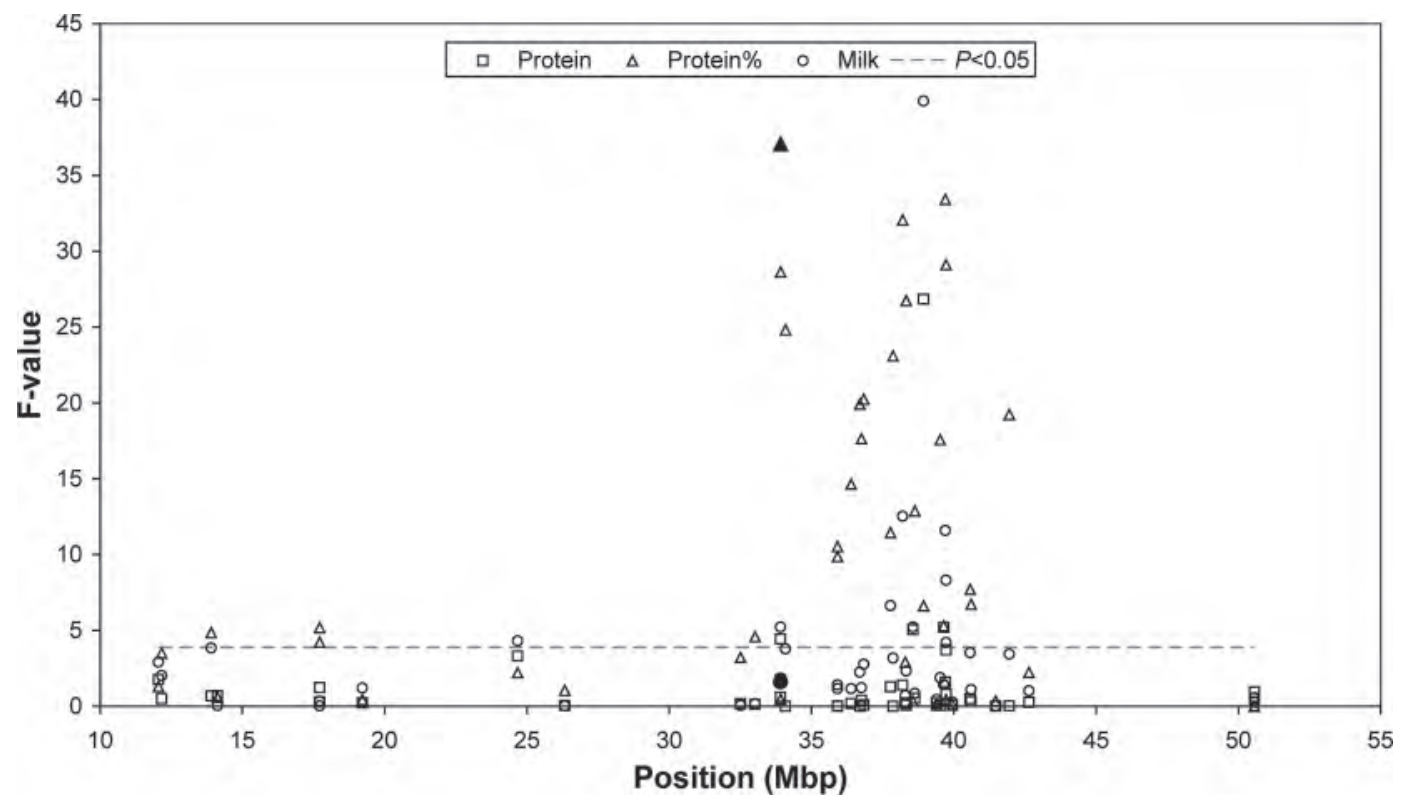

Figure 1. Single SNP regression results of all validated SNP on chromosome 20 for protein and milk yields and protein composition. The enlarged solid data point at $33 \mathrm{Mb}$ in each series is the published mutation in growth hormone receptor (Blott et al., 2003). 
Table 4. Linkage disequilibrium $\left(\mathrm{r}^{2}\right)$ between all 13 significant $\mathrm{SNP}^{1}$ on chromosome 20 from the multi-SNP analysis on the off-diagonal and SNP position within genome build Btau4.2 on the diagonal

\begin{tabular}{|c|c|c|c|c|c|c|c|c|c|c|c|c|c|}
\hline & 1 & 2 & 3 & 4 & 5 & 6 & 7 & 8 & 9 & 10 & 11 & 12 & 13 \\
\hline 1 & $14,123,347$ & 0.47 & 0.15 & 0.25 & 0.14 & 0.10 & 0.05 & 0.07 & 0.00 & 0.09 & 0.09 & 0.07 & 0.06 \\
\hline 2 & & $17,715,492$ & 0.17 & 0.13 & 0.19 & 0.19 & 0.16 & 0.17 & 0.02 & 0.11 & 0.01 & 0.15 & 0.01 \\
\hline 3 & & & $24,676,083$ & 0.17 & 0.07 & 0.20 & 0.06 & 0.07 & 0.05 & 0.09 & 0.02 & 0.09 & 0.08 \\
\hline 4 & & & & $26,333,982$ & 0.26 & 0.25 & 0.19 & 0.16 & 0.21 & 0.20 & 0.21 & 0.19 & 0.25 \\
\hline 5 & & & & & $32,505,231$ & 0.49 & 0.21 & 0.08 & 0.31 & 0.27 & 0.34 & 0.05 & 0.14 \\
\hline 6 & & & & & & $33,918,868$ & 0.49 & 0.40 & 0.64 & 0.54 & 0.53 & 0.40 & 0.29 \\
\hline 7 & & & & & & & $37,776,292$ & 0.30 & 0.67 & 0.56 & 0.50 & 0.33 & 0.57 \\
\hline 8 & & & & & & & & $37,869,051$ & 0.44 & 0.68 & 0.43 & 0.85 & 0.34 \\
\hline 9 & & & & & & & & & $38,204,592$ & 0.79 & 0.74 & 0.53 & 0.44 \\
\hline 10 & & & & & & & & & & $38,328,800$ & 0.66 & 0.65 & 0.46 \\
\hline 11 & & & & & & & & & & & $39,642,805$ & 0.44 & 0.41 \\
\hline 12 & & & & & & & & & & & & $39,701,308$ & 0.36 \\
\hline 13 & & & & & & & & & & & & & 40,005 \\
\hline
\end{tabular}

rs41581070; $13=$ rs29016210; SNP 6 does not have an rs number.

Table 5. Single nucleotide polymorphism effects for Australian Selection Index (ASI), protein yield, protein percentage, fat yield, fat percentage, and milk yield for 6 SNP (corresponds to Table 4) from the final multiple SNP analysis and their significance

\begin{tabular}{|c|c|c|c|c|c|c|c|}
\hline $\begin{array}{l}\text { refSNP } \\
\text { entry }\end{array}$ & $\begin{array}{l}\text { Position } \\
\text { (bp) }\end{array}$ & ASI & $\begin{array}{l}\text { Protein } \\
\text { yield }(\mathrm{kg})\end{array}$ & $\begin{array}{c}\text { Protein } \\
\text { percentage (\%) }\end{array}$ & $\begin{array}{l}\text { Fat yield } \\
(\mathrm{kg})\end{array}$ & $\begin{array}{c}\text { Fat percentage } \\
(\%)\end{array}$ & $\begin{array}{l}\text { Milk yield } \\
\quad(\mathrm{kg})\end{array}$ \\
\hline rs 29013325 & $17,715,492$ & $7.34 \pm 2.1^{* * *}$ & $0.03 \pm 0.79$ & $0.0272 \pm 0.008^{* * *}$ & $2.96 \pm 1.13^{*}$ & $0.0725 \pm 0.019^{* * *}$ & $-57 \pm 38$ \\
\hline rs29026076 & $24,676,083$ & $-3.70 \pm 5.81$ & $-2.61 \pm 2.13$ & $0.0433 \pm 0.022 *$ & $-4.22 \pm 3.06$ & $0.0439 \pm 0.053$ & $-186 \pm 103$ \\
\hline rs29022923 & $26,333,982$ & $4.38 \pm 2.31$ & $-0.05 \pm 0.84$ & $0.0060 \pm 0.009$ & $3.67 \pm 1.22^{* *}$ & $0.0587 \pm 0.021^{* *}$ & $-20 \pm 40$ \\
\hline & $33,918,868^{1}$ & $-7.73 \pm 7.55$ & $-1.33 \pm 2.80$ & $-0.0276 \pm 0.028$ & $-1.85 \pm 3.98$ & $-0.0362 \pm 0.068$ & $-4 \pm 136$ \\
\hline rs41257066 & $38,204,592$ & $-1.24 \pm 2.82$ & $0.62 \pm 1.04$ & $-0.0317 \pm 0.010^{* *}$ & $-0.72 \pm 1.49$ & $-0.0632 \pm 0.026^{*}$ & $86 \pm 50$ \\
\hline rs41581070 & $39,701,308$ & $-4.36 \pm 3.12$ & $0.79 \pm 1.15$ & $-0.0462 \pm 0.012^{* * *}$ & $-1.83 \pm 1.64$ & $-0.0994 \pm 0.028^{* * *}$ & $121 \pm 55^{* * *}$ \\
\hline
\end{tabular}

Growth hormone receptor $($ GHR $)$ SNP published by Blott et al. (2003)

○ $\quad * * * P<0.001 ;{ }^{* *} P<0.01 ;{ }^{*} P<0.05$. 
to affect fat yield and could therefore be detecting the same QTL, even though each SNP is also associated with other traits, which could indicate more than one QTL in this region. The GHR SNP published by Blott et al. (2003) does not appear to be significantly associated with any of the 6 traits; however, it does have the largest effect on ASI and a large effect on protein yield. The SNP at 38,204,592 and 39,701,308 bp both appear to increase milk yield and decrease protein and fat composition and so could be detecting the same QTL.

\section{DISCUSSION}

Table 1 summarizes the results from each of the SNP screening experiments. For all of these experiments, we observed FDR $<100 \%$, suggesting that at least some of them are real associations. The only reliable way to confirm these associations is to validate the results in a different population (Lander and Kruglyak, 1995; Visscher, 2008). Therefore, $423 \mathrm{SNP}$ were selected from the screening experiments and genotyped across 411 additional Holstein bulls from Genetics Australia's 2001-2003 progeny test teams.

Two hundred eighty-nine SNP, $68 \%$, from this validation data set were polymorphic in the validation population and passed quality checks. Several SNP failed assay design at the assay design and genotyping steps, particularly GWAS SNP originally genotyped on the commercial SNP chips. The SNP from the CGS experiment were originally genotyped using the same SNPlex chemistry and so were more successful in the new SNPlex pools. Each SNP was tested for 6 traits. Table 2 summarizes the validation results for each of the 6 traits: $25,23,47,25,38$, and 28 markers were significant $(P<0.05)$ for ASI, protein yield, protein composition, fat yield, fat composition, and milk yield, respectively, where 14 significant results per trait would be expected by chance alone. We can see that the proportion of significant SNP that were actually validated (significant at $P<0.05$ and the SNP effect in the same direction as in the screening experiment) was high for ASI when all SNP from the validation were included, but also when only SNP significant for ASI in the screening experiment were included. This is because the selection criteria from all screening experiments focused on ASI; therefore, the majority of SNP were significantly associated with ASI in the screening experiment, resulting in little difference between FDR and FDR $\mathrm{a}_{\mathrm{a}}$. The proportion of significant SNP that were actually validated for protein was low, even when only those SNP significant in the screening experiment were used. This could be a result of the size of the QTL and therefore the power of the experiments to detect them. It has been suggested that the size of individual
QTL affecting protein yield is small (Chamberlain et al., 2007). For traits other than ASI and protein yield, FDR and $F R_{a}$ improved when only SNP that were significant in the screening experiment were included, as expected. Overall, 113 CGS and 176 GWAS SNP were found, with 12 and 23 significant for ASI, resulting in similar proportions of significant SNP, 11 and $13 \%$, respectively. However, the proportion of these significant SNP that had SNP effects in the same direction as in the screening experiment was higher for CGS SNP (83\%) compared with that for GWAS SNP (65\%). Overall, the validation rate for SNP from CGS was not any better than that for GWAS.

The FDR for the validated SNP was calculated in 2 ways, the first being the accepted method of Benjamini and Hochberg (1995) and based on the total number of tests, significant tests, and the significance threshold. The second method, FDR , was based on the proportion of significant SNP with SNP effects in the same direction as in the screening experiment; that is, the proportion of significant SNP validated. When the observed proportion of validated SNP was $<50 \%$, the $\mathrm{FDR}_{\mathrm{a}}$ returned $100 \%$. This is because the calculation assumes that SNP that are not true QTL (i.e., false positives) will have SNP effects that are random and therefore will be in the same direction as the screening experiment $50 \%$ of the time by chance alone.

Table 3 presents the 72 SNP that were validated, 36 of which were selected from multiple studies. The table also identifies 30 SNP that were originally selected as part of the CGS. These SNP were selected because they were within or close to genes with a function that could be associated with milk synthesis in dairy cattle. These candidate genes were located within 26 regions found to be associated with ASI in the $10 \mathrm{~K}$ experiment. These 30 SNP fall within 12 of those 26 regions. As a further validation, Table 3 also presents the distance between the SNP itself and the most significant SNP, within 5 Mb, from a GWAS utilizing 39,048 SNP (Pryce et al., 2010a). When no distance is listed, no significant SNP was found from the $50 \mathrm{~K}$ experiment within $5 \mathrm{Mb}$. In total, 51,386 SNP were genotyped across 1,530 bulls from 2 breeds; 39,048 SNP were polymorphic, passed genotype quality control, and were tested for association with 7 traits (the same 6 traits used here and female fertility). Although the power of that experiment was not 1 , it was much more powerful than any of the experiments presented here and thus it would be expected that most of the associations found in this study would be detected. Ninety-six percent of $10-\mathrm{Mb}$ intervals were found to have a significant SNP for any of the 6 traits in the $50 \mathrm{~K}$ experiment. Of the $67 \mathrm{SNP}$ with known positions in the present experiment, 56 $\mathrm{SNP}$ were within $5 \mathrm{Mb}$ of a significant SNP from the 
$50 \mathrm{~K}$ experiment. If $96 \%$ of all $10-\mathrm{Mb}$ intervals had a significant SNP in the $50 \mathrm{~K}$ experiment, then 56 is less than the 64 expected. This means that some of the associations validated here were not detected in the 50K experiment. These may be false positives, although this is unlikely considering they have been validated in 2 different populations. Another explanation could be that the $50 \mathrm{~K}$ experiment has few SNP in those areas of the genome. On closer inspection of the 50K experiment results, the SNP with associations validated here and not detected in the $50 \mathrm{~K}$ experiment all have at least one SNP within the mean distance between SNP (76,828 bp), some of which are present in the 39,048 SNP tested in the experiment. Some of these SNP do show associations with other milk production traits not validated here. The only remaining explanation is that the $50 \mathrm{~K}$ experiment failed to detect all associations.

The correlation between ASI and protein composition was positive; that is, an animal with a high protein composition breeding value also had a high ASI breeding value. This is because ASI has a positive weighting on protein yield and a negative weighting on milk yield. The correlation between SNP effects for ASI and protein composition for all SNP and for only the SNP significant for both traits was calculated as 0.21 and 0.9 , respectively. It shows that the direction and magnitude of the SNP effects for ASI and protein composition when using SNP from the whole data set were lowly correlated; that is, some would be in the same direction just by chance, and a large SNP effect for ASI does not always mean that the SNP effect for protein composition will be large. However, it also shows that when only SNP significant for both traits were considered, SNP effects for ASI and protein composition were always in the same direction and of a similar magnitude and therefore more likely to be true effects.

A mutation in the GHR gene on chromosome 20 has been published as having an effect on protein composition, and protein and milk yields (Blott et al., 2003). This SNP was genotyped and found to be associated with milk production traits in the CGS experiment and was therefore included in this validation data set. The association between the published GHR SNP and protein composition was validated in this population. Figure 1 presents the results of the single SNP regression analysis for all SNP on chromosome 20 for protein composition and protein and milk yields. It shows that the published GHR mutation at $33 \mathrm{Mb}$ was only associated with protein composition in this population; no significant association was found with milk and protein yields. Another SNP at $39 \mathrm{Mb}$ was, however, associated with milk and protein yields. A multiple SNP regression analysis was performed for all 6 milk production traits to see if all SNP were picking up the association with the published $G H R$ mutation or whether multiple QTL were present on this chromosome.

Although the final multiple SNP regression model consisted of 13 SNP, some of these SNP were highly correlated and most likely detecting the same QTL. Therefore, this SNP set was reduced to 6 by excluding SNP highly correlated with others in the set of 13, starting with SNP fitted last in the model. The final multi-SNP analysis, which included the Blott et al. (2003) SNP in the GHR gene, then revealed that this mutation had the largest effect on ASI and a large effect on protein yield; however, it was not significantly associated with any of the milk production traits. However, at least 2 other QTL were present on chromosome 20. The first QTL was detected by 3 SNP at $17,715,492,24,676,083$, and $26,333,982 \mathrm{bp}$; all 3 had an effect on fat yield. However, other associations that were not consistent across the 3 SNP were detected, so it is possible that more than one QTL exists in that region. According to bovine genome build Btau4.2, 47 genes are located between 17 and $27 \mathrm{Mb}$, so further fine mapping of this region would be required to determine any causative genes or mutations. The second QTL was detected by 2 SNP at $38,204,592$ and 39,701,308 bp; the SNP effects for both of these SNP are consistent across the 6 milk production traits with the QTL increasing milk yield and decreasing protein and fat composition. According to bovine genome build Btau4.2, 11 genes are between 38 and 40 Mb. The SNP at 38,204,592 bp flanks a gene called leukemia inhibitory factor receptor $(L I F R)$. The SNP at $39,701,308 \mathrm{bp}$, which is most highly associated with the 3 traits, is located close to a gene called glial cellderived neurotrophic factor $(G D N F)$. Leukemia inhibitory factor receptor s known to be involved in mammary gland apoptosis (Schere-Levy et al., 2003), and GDNF encodes a highly conserved neurotrophic factor that potently promotes the survival of many types of neurons (Lin et al., 1993). Mutations in this gene may be associated with Hirschsprung disease (Eketjall and Ibanez, 2002), a common congenital abnormality leading to bowel obstructions in humans. Mutations in this gene in dairy cattle could result in reduced uptake of nutrients from the gut. Alternatively, although not demonstrated in humans, this gene could have a similar effect on other cell types including neural cells of the mammary gland or even mammary epithelial cells. Interestingly, the mutation in the prolactin receptor gene $(P R L R)$ on chromosome 20, noted by Viitala et al. (2006) to have an effect on milk, fat, and protein production, was tested but not confirmed in this data set.

\section{CONCLUSIONS}

Out of the 423 SNP that were tested in our validation population, 72 were significantly associated with milk 
production traits. A number of these SNP were either in or close to genes that have not previously been reported to affect milk production in dairy cattle, such as LIFR and GDNF. However, an over-representation of SNP (43 of the 423) on chromosome 20 was observed, including a SNP in the growth hormone receptor gene that was previously reported to be associated with protein percentage. When a multiple SNP regression analysis for all SNP on chromosome 20 was performed for all 6 traits, this mutation was not significantly associated with any of the milk production traits; however, there was evidence that at least 2 other QTL were present on chromosome 20.

\section{ACKNOWLEDGMENTS}

The authors acknowledge the work of Thamy Balasingham, Catriona Thompson, Hayley Mountford, Sally Potter, Jodi Ryan, and Julie Penninckx (Department of Primary Industries Victoria, Australia) for the genotyping, sequencing, and SNP detection involved in this project. The samples were provided by Genetics Australia (Bacchus Marsh, Victoria, Australia), and the phenotypes were provided by the Australian Dairy Herd Improvement Scheme (Melbourne, Australia). Finally, the project was funded by the Victorian State Governments Our Rural Landscape initiative.

\section{REFERENCES}

Benjamini, Y., and Y. Hochberg. 1995. Controlling the false discovery rate - A practical and powerful approach to multiple testing. J. R. Stat. Soc. B 57:289-300.

Blott, S., J. J. Kim, S. Moisio, A. Schmidt-Kuntzel, A. Cornet, P. Berzi, N. Cambisano, C. Ford, B. Grisart, D. Johnson, L. Karim, P. Simon, R. Snell, R. Spelman, J. Wong, J. Vilkki, M. Georges, F. Farnir, and W. Coppieters. 2003. Molecular dissection of a quantitative trait locus: A phenylalanine-to-tyrosine substitution in the transmembrane domain of the bovine growth hormone receptor is associated with a major effect on milk yield and composition. Genetics 163:253-266.

Bovine HapMap Consortium. 2009. Genome-wide survey of SNP variation uncovers the genetic structure of cattle breeds. Science 324:528-532.

Chamberlain, A. J., H. C. McPartlan, and M. E. Goddard. 2007. The number of loci that affect milk production traits in dairy cattle. Genetics 177:1117-1123.

Eketjall, S., and C. F. Ibanez. 2002. Functional characterization of mutations in the GDNF gene of patients with Hirschsprung disease. Hum. Mol. Genet. 11:325-329.

Elsik, C. G., R. L. Tellam, K. C. Worley, and C. Bovine Genome Sequencing Anal. 2009. The genome sequence of taurine cattle: A window to ruminant biology and evolution. Science 324:522-528.

Gilmour, A., B. Cullis, R. Welham, and R. Thompson. 2001. ASREML Reference Manual. New South Wales Department of Primary Industries, Sydney, Australia.

Hardenbol, P., J. Baner, M. Jain, M. Nilsson, E. A. Namsaraev, G. A. Karlin-Neumann, H. Fakhrai-Rad, M. Ronaghi, T. D. Willis, U. Landegren, and R. W. Davis. 2003. Multiplexed genotyping with sequence-tagged molecular inversion probes. Nat. Biotechnol. 21:673-678.
Henderson, C. R. 1975. Best linear unbiased estimation and prediction under a selection model. Biometrics 31:423-447.

Karlsson, E. K., I. Baranowska, C. M. Wade, N. H. C. Salmon Hillbertz, M. C. Zody, N. Anderson, T. M. Biagi, N. Patterson, G. R. Pielberg, E. J. Kulbokas, K. E. Comstock, E. T. Keller, J. P. Mesirov, H. von Euler, O. Kampe, A. Hedhammar, E. S. Lander, R. Andersson, L. Andersson, and K. Lindblad-Toh. 2007. Efficient mapping of mendelian traits in dogs through genome-wide association. Nat. Genet. 39:1321-1328.

Lander, E., and L. Kruglyak. 1995. Genetic dissection of complex traits-Guidelines for interpreting and reporting linkage results. Nat. Genet. 11:241-247.

Lin, L. F. H., D. H. Doherty, J. D. Lile, S. Bektesh, and F. Collins. 1993. GDNF - A glial-cell line derived neurotrophic factor for midbrain dopaminergic-neurons. Science 260:1130-1132.

Mosig, M. O., E. Lipkin, G. Khutoreskaya, E. Tchourzyna, M. Soller, and A. Friedmann. 2001. A whole genome scan for quantitative trait loci affecting milk protein percentage in Israeli-Holstein cattle, by means of selective milk DNA pooling in a daughter design, using an adjusted false discovery rate criterion. Genetics 157:1683-1698.

Naukkarinen, J., I. Surakka, K. H. Pietilainen, A. Rissanen, V. Salomaa, S. Ripatti, H. Yki-Jarvinen, C. M. van Duijn, H. E. Wichmann, J. Kaprio, M. R. Taskinen, and L. Peltonen., and the ENGAGE Consortium. 2010. Use of genome-wide expression data to mine the "gray zone" of GWA studies leads to novel candidate obesity genes. PLoS Genet. 6:e1000976.

Pryce, J. E., S. Bolormaa, A. J. Chamberlain, P. J. Bowman, K. Savin, M. E. Goddard, and B. J. Hayes. 2010a. A validated genomewide association study in 2 dairy cattle breeds for milk production and fertility traits using variable length haplotypes. J. Dairy Sci. 93:3331-3345.

Pryce, J. E., J. van der Werf, M. Haile-Mariam, B. Malcolm, and M. E. Goddard. 2010b. A technical manual describing the Australian Profit Ranking (APR) index. Version 2. December 2010. Accessed Dec. 15, 2010. http://www.adhis.com.au/.

Pryce, J. E., J. H. J. van der Werf, M. Haile-Mariam, B. Malcolm, and M. E. Goddard. 2009. Updated index weights for the Australian profit ranking in dairy cattle. Proc. Assoc. Adv. Anim. Breed. Genet. 18:143-146.

Scheet, P., and M. Stephens. 2006. A fast and flexible statistical model for large-scale population genotype data: Applications to inferring missing genotypes and haplotypic phase. Am. J. Hum. Genet. 78:629-644.

Schere-Levy, C., V. Buggiano, A. Quaglino, A. Gattelli, M. C. Cirio, I. Piazzon, S. Vanzulli, and E. C. Kordon. 2003. Leukemia inhibitory factor induces apoptosis of the mammary epithelial cells and participates in mouse mammary gland involution. Exp. Cell Res. 282:35-47.

Storey, J. D., and R. Tibshirani. 2003. Statistical significance for genomewide studies. Proc. Natl. Acad. Sci. USA 100:9440-9445.

Viitala, S., J. Szyda, S. Blott, N. Schulman, M. Lidauer, A. MakiTanila, M. George, and J. Vilkki. 2006. The role of the bovine growth hormone receptor and prolactin receptor genes in milk, fat and protein production in Finnish Ayrshire dairy cattle. Genetics 173:2151-2164.

Visscher, P. M. 2008. Sizing up human height variation. Nat. Genet. 40:489-490.

Weller, J. I., J. Z. Song, D. W. Heyen, H. A. Lewin, and M. Ron. 1998. A new approach to the problem of multiple comparisons in the genetic dissection of complex traits. Genetics 150:1699-1706.

\section{APPENDIX}

\section{Deregressing Bull Breeding Values}

Deregressed breeding values $(\wp)$ provide the equivalent of LS estimates of the animals breeding values, adjusted for fixed effects, for the trait of interest. That 
is, they can be modeled as $\wp=a+e$, where $a$ is the breeding value, $e$ is the error, and the covariance between $a$ and $e$ is equal to zero. Australian Breeding Values were deregressed using the method presented here. The ADHIS calculated ABV for bulls in a series of single-trait, animal model BLUP equations (Henderson, 1975). The deregression method mimics the analysis by assuming that the ABV for each bull was based on $N_{i}$ independent phenotypic records from his daughters (effective number of daughters for bull $i$ ), corrected for fixed effects. These records $(y)$ were modeled as $y=$ $\mathbf{Z} \mathbf{s}+e$, where $\mathbf{s}$ is a vector of sire effects, $\mathbf{Z}$ is a design matrix that relates the phenotypes to the sires, and $e$ is the error, which is assumed to be $\sim \mathrm{N}\left(0, \sigma^{2}\right)$. This led to BLUP equations $\left(\mathbf{Z}^{\prime} \mathbf{Z}+\mathbf{A}^{-1} \lambda\right) \hat{s}=\mathbf{Z}^{\prime}(y)$, where $\mathbf{Z} \mathbf{Z}$ is a diagonal matrix of elements $N_{i}$, $\mathbf{A}$ is the numerator relationship matrix among all sires based on sire/maternal grandsire pedigree, and $\lambda=\left(4-h^{2}\right) / h^{2}$, where $h^{2}$ is the heritability of the trait. These equations can also be considered an approximation to the ADHIS BLUP equations when all fixed effects and all other animal effects have been absorbed. It is $\bar{y}_{i}$, the average of a sire's daughters, that is needed, but the right-hand side of the above equations is unknown. However, the lefthand side can be constructed and hence $\left(\mathbf{Z}^{\prime} \mathbf{Z}+\mathbf{A}^{-1} \lambda\right) \hat{s}$ can be calculated to yield $\mathbf{Z}^{\prime}(y)$. The values of $\hat{s}$ and their corresponding reliabilities $(R)$ were provided by ADHIS, and the reliability of $\hat{s}_{i}\left(R_{i}\right)$ in the equations above is given by $\left(\mathbf{Z}^{\prime} \mathbf{Z}+\mathbf{A}^{-1} \lambda\right)_{i i}^{-1}=\left(1-R_{i}\right) / \lambda$. Therefore, the values of $N_{i}$ were adjusted in an iterative approach until this calculation agreed with the reliabilities provided by ADHIS for all animals. However, ADHIS was not exact in its calculation of $R$ and so the values of $N$ based on this value of $R$ were also inexact. $\bar{y}_{i}$, the average of a sires daughters, $\frac{\left(\mathbf{Z}^{\prime} y\right)_{i}}{n_{i}}$, was calculated, where $n$ is the number of daughters for sire $i$, and $\wp=2 \bar{y}_{i}$ was used as the final phenotype as sire effects are half breeding values; that is, $\hat{s}=\frac{\hat{a}}{2}$.

\section{Deregressing Cow Breeding Values}

Deregressing breeding values provided an estimate of an animal's average phenotypic yield, adjusted for fixed effects, for the trait of interest, over the animal's lifetime. The ABV were deregressed using the method presented here. The ADHIS calculated ABV for cows and bulls in a series of single-trait, animal model BLUP equations (Henderson, 1975). The deregression method mimics the analysis by assuming that the ABV for a cow is based on $N_{c}$ independent phenotypic records on the cow, corrected for fixed effects, $N_{s}$ records on her sire, $N_{d}$ records on her dam, and $N_{m}$ records on her maternal grandsire. Because males cannot have milk production records, these records on the sire and maternal grandsire mimic the information on these bulls coming from other relatives. These records $(y)$ can be modeled as $y=\mathbf{Z a}+e$, where $\mathbf{a}$ is a vector of breeding values, $\mathbf{Z}$ is a design matrix that relates the phenotypes to the estimated breeding values, and $e$ is the error, which is assumed to be $\sim \mathrm{N}\left(0, \sigma^{2}\right)$. This leads to BLUP equations $\left(\mathbf{Z}^{\prime} \mathbf{Z}+\mathbf{A}^{-1} \lambda\right) \hat{a}=\mathbf{Z}^{\prime}(y)$, where $\mathbf{A}$ is the numerator relationship matrix and $\lambda=\left(1-h^{2}\right) / h^{2}$, where $h^{2}$ is the heritability of the trait. Written in full, the equations are

$$
\left[\begin{array}{cccc}
N_{c}+2 \lambda & -\lambda & -\lambda & 0 \\
-\lambda & N_{s}+1.5 \lambda & 0.5 \lambda & 0 \\
-\lambda & 0.5 \lambda & N_{d}+1.833 \lambda & -2 / 3 \lambda \\
0 & 0 & -2 / 3 \lambda & N_{m}+1.33 \lambda
\end{array}\right]\left[\begin{array}{c}
\hat{c} \\
\hat{s} \\
\hat{d} \\
\hat{m}
\end{array}\right]=\left[\begin{array}{c}
N_{c} y_{c} \\
N_{s} y_{s} \\
N_{d} y_{d} \\
N_{m} y_{m}
\end{array}\right] \text {. }
$$

These equations are considered an approximation to the ADHIS BLUP equations when all fixed effects and all other animal effects have been absorbed. It is $y_{c}$, the lifetime average yield of the cow, that is needed, but the right-hand side of the above equation is unknown. However, the left-hand side can be constructed and hence the calculation of $\left(\mathbf{Z}^{\prime} \mathbf{Z}+\mathbf{A}^{-1} \lambda\right) \hat{a}=\mathbf{Z}^{\prime} y$ solved. The values of $\hat{a}(\mathrm{ABV})$ and their corresponding reliabilities $(R)$ were provided by ADHIS, and the reliability of $\hat{a}_{i}\left(R_{i}\right)$ in the equations above is $\left(\mathbf{Z}^{\prime} \mathbf{Z}+\mathbf{A}^{-1} \lambda\right)_{i i}^{-1}=\left(1-R_{i}\right) / \lambda$. Therefore, the values of $N_{i}$ were adjusted in an iterative approach until this calculation agreed with the reliabilities provided by ADHIS for all 4 animals. The ADHIS was not exact in its calculation of $R$ and so the values of $N$ based on this value of $R$ were inexact. If a dam was unknown but the maternal grandsire was known, $N_{d}$ was fixed to 0 and $\hat{d}$ was calculated. If both dam and maternal grandsire were unknown, $N_{d}$ and $N_{m}$ were fixed to 0 and $\hat{d}$ calculated. Once the values of $N$ were known, $\bar{y}_{c}=\frac{\left(N_{c}+2 \lambda\right) \hat{c}-\lambda \hat{s}-\lambda \hat{d}}{N_{c}}$ was calculated. Relatives were included in the estimation of $\bar{y}_{c}$ because they were used in the calculation of $\mathrm{ABV}$ and $R$. Deregressed breeding values were then corrected for the sire and dam breeding values, $\bar{y}_{c}-0.5 \hat{s}-0.5 \hat{d}$, and $N_{c}$ was used as a weight in the analysis. 